\title{
The Pluripolar Hull and the Fine Topology
}

by

\author{
Armen EDIGARIAN
}

Presented by Józef SICIAK

Summary. We show that the projections of the pluripolar hull of the graph of an analytic function in a subdomain of the complex plane are open in the fine topology.

1. Introduction. Let $\Omega \subset \mathbb{C}^{n}$ be an open set and let $E \subset \Omega$ be any subset. We say that $E$ is pluripolar in $\Omega$ if for all $z \in E$ there exist a connected neighborhood $U_{z}$ of $z$ in $\Omega$ and a plurisubharmonic function $u(z, w) \not \equiv-\infty$ defined on $U_{z}$ such that

$$
E \cap U_{z} \subset\left\{(z, w) \in U_{z}: u(z, w)=-\infty\right\} .
$$

By Josefson's theorem (see [Jos]), a set $E \subset \mathbb{C}^{N}$ is pluripolar if and only if there exists a globally defined plurisubharmonic function $u(z, w)$ such that

$$
E \subset\left\{(z, w) \in \mathbb{C}^{N}: u(z, w)=-\infty\right\} .
$$

By the pluripolar hull $E_{\Omega}^{*}$ (see [LePo]) of a pluripolar subset $E \subset \Omega$, we mean

$$
E_{\Omega}^{*}:=\bigcap\{z \in \Omega: u(z)=-\infty\},
$$

where the intersection is taken over all plurisubharmonic functions $u$ in $\Omega$ which equal $-\infty$ on $E$. In general, it is difficult to describe the pluripolar hull of a given set $E$. The following theorem, recently proved in [EdWi3], gives some information about $E_{\Omega}^{*}$.

THEOREM 1. Let $\Omega$ be a pseudoconvex open set in $\mathbb{C}^{N}$ and let $E \subset \Omega$ be an $F_{\sigma}$ pluripolar subset. If $E$ is connected then so is $E_{\Omega}^{*}$.

The following main result of the paper gives another property of $E_{\Omega}^{*}$.

2000 Mathematics Subject Classification: 31C40, 32U05.

Key words and phrases: pluripolar set, pluripolar hull, thin point, fine topology.

Research partially supported by KBN Grant No. 1PO3A 00528 . The author is a fellow of Krzyżanowski Fund at the Jagiellonian University. 
THEOREM 2. Let $\Omega$ be a pseudoconvex open set in $\mathbb{C}^{N}$ and let $E \subset \Omega$ be an $F_{\sigma}$ pluripolar subset. Assume that $U \subset \Omega$ is an open neighborhood of $E_{\Omega}^{*}$ and that $f: U \rightarrow \mathbb{C}$ is a non-constant holomorphic function. Then for any $p \in f\left(E_{\Omega}^{*}\right) \backslash f(E)$ the set $\mathbb{C} \backslash f\left(E_{\Omega}^{*}\right)$ is thin at $p$.

Moreover, if $f(E)$ is open in the fine topology then so is $f\left(E_{\Omega}^{*}\right)$.

A set $F \subset \mathbb{C}$ is thin at a point $\xi$ if either $\xi \notin \bar{F}$, or $\xi \in \bar{F}$ and there exists a subharmonic function $h$ in a neighborhood of $\xi$ such that $\varlimsup_{z \in F, z \rightarrow \xi} h(z)<$ $h(\xi)$. One can always choose $h$ in such a way that the upper limit equals $-\infty$ (see e.g. [Ran]).

Starting from a paper of Sadullaev [Sad] the pluripolar hull of graphs of certain analytic functions has been studied in a number of papers (see e.g. [EdWi1]-[EdWi3], [E-J2], [LePo], [Sic], [Wie1], [Wie2], and [Zwo]).

For a subset $A$ of the complex plane $\mathbb{C}$ and a complex-valued function $f$ on $A$ we denote by $\Gamma_{f}(A)$ the graph of $f$ over $A$,

$$
\Gamma_{f}(A)=\left\{(z, w) \in \mathbb{C}^{2}: z \in A, w=f(z)\right\} .
$$

Let $f$ be a holomorphic function in a domain $D \subset \mathbb{C}$. It is immediate that $\Gamma_{f}(D)$ is a pluripolar set. Supported by several examples, in [LeMaPo] Levenberg, Martin and Poletsky conjectured that if $f$ is analytic in $D$ and the domain of existence of $f$ is $D$, then $\Gamma_{f}(D)$ is complete pluripolar. This conjecture was disproved in [EdWi2] (in case of the unit disc) and in [EdWi1] (in case of a domain $D=\mathbb{C} \backslash K$, where $K$ is a compact polar set).

Denote by $\pi_{j}$ the projection onto the $j$ th coordinate plane in $\mathbb{C}^{2}, \pi_{j}(z)=$ $z_{j}$ for $z=\left(z_{1}, z_{2}\right) \in \mathbb{C}^{2}, j=1,2$. As a corollary of Theorem 2 we get the following result, which is a positive answer to Problem 1 posed in [E-J1] (in a revised version [E-J2], the authors get independently the first part of the corollary).

Corollary 3. Let $D \subset \mathbb{C}$ be a domain and let $f$ be an analytic function in $D$. Then $\pi_{1}\left(\left(\Gamma_{f}(D)\right)_{\mathbb{C}^{2}}^{*}\right)$ is open in the fine topology. Moreover, if $f$ is non-constant then $\pi_{2}\left(\left(\Gamma_{f}(D)\right)_{\mathbb{C}^{2}}^{*}\right)$ is also open in the fine topology.

The fine topology is the weakest topology for which all subharmonic functions are continuous. A neighborhood basis of a point in this topology consists of sets which differ from a Euclidean neighborhood of this point by a set which is thin at this point (see e.g. [Bre]). Hence, if $A \subset \mathbb{C}$ is any set then $A$ is open in the fine topology if and only if $\mathbb{C} \backslash A$ is thin at each $p \in A$.

2. Preliminary results. Let $\Omega$ be a domain in $\mathbb{C}^{N}$. In [LePo] the negative pluripolar hull is defined as

$$
E_{\Omega}^{-}:=\bigcap\{z \in \Omega: u(z)=-\infty\},
$$


where the intersection is taken over all negative plurisubharmonic functions $u$ in $\Omega$ that are $-\infty$ on $E$. The following relation between the negative pluripolar hull and the pluripolar hull holds (see [LePo]).

ThEOREM 4. Let $\Omega$ be a pseudoconvex domain in $\mathbb{C}^{N}$. Let $\left\{\Omega_{j}\right\}$ be an increasing sequence of relatively compact subdomains of $\Omega$ with $\bigcup_{j} \Omega_{j}=\Omega$. Let $E \subset \Omega$ be pluripolar. Then

$$
E_{\Omega}^{*}=\bigcup_{j}\left(E \cap \Omega_{j}\right)_{\Omega_{j}}^{-} .
$$

For a subset $E \subset \Omega$, the pluriharmonic measure at a point $z \in \Omega$ of $E$ relative to $\Omega$ is defined as

$$
\begin{aligned}
& \omega(z, E, \Omega) \\
& \quad=-\sup \left\{u(z): u \text { is plurisubharmonic in } \Omega \text { and } u \leq-\chi_{E}\right\},
\end{aligned}
$$

where $\chi_{E}$ is the characteristic function of $E$. The relation between the negative pluripolar hull and the pluriharmonic measure is given in the following theorem (see [LePo]).

ThEOREM 5. Let $\Omega$ be a domain in $\mathbb{C}^{N}$ and let $E \subset \Omega$ be pluripolar. Then

$$
E_{\Omega}^{-}=\{z \in \Omega: \omega(z, E, \Omega)>0\} .
$$

From Theorem 5 we get the following

Corollary 6. Let $\Omega$ be a pseudoconvex domain in $\mathbb{C}^{N}$ and let $E \subset \Omega$ be an $F_{\sigma}$ pluripolar subset. Then $E_{\Omega}^{*}$ is also an $F_{\sigma}$ set.

Proof. Let $E=\bigcup_{j} K_{j}$, where $K_{1} \subset K_{2} \subset \cdots$ are compact sets. Then $E_{\Omega}^{*}=\bigcup_{j}\left(K_{j}\right)_{\Omega}^{*}$. So, it sufficient to show that $K_{\Omega}^{*}$ is an $F_{\sigma}$ set for any compact pluripolar set $K$. Take an increasing sequence of relatively compact hyperconvex domains $\Omega_{j}$ so that $K \subset \Omega_{1}$ and that $\Omega=\bigcup_{j} \Omega_{j}$. Then $K_{\Omega}^{*}=\bigcup_{j=1}^{\infty} K_{\Omega_{j}}^{-}$and $K_{\Omega_{j}}^{-}=\bigcup_{k=1}^{\infty}\left\{z \in \Omega_{j}: \omega\left(z, K, \Omega_{j}\right) \geq 1 / k\right\}$. Recall that $\omega\left(\cdot, K, \Omega_{j}\right)$ is an upper semicontinuous function.

The following result is well known. For the sake of completeness we give the proof.

Proposition 7. Let $E$ be a Borel polar set in $\mathbb{C}$. Then $E_{\mathbb{C}}^{*}=E$.

Proof. Fix $z_{0} \notin E$. By Choquet's theorem there exists a sequence of open sets $U_{1} \supset U_{2} \supset \cdots \supset E$ such that $z_{0} \notin U_{j}$ and $c\left(U_{j}\right) \rightarrow 0$ when $j \rightarrow \infty$. Here $c$ is the logarithmic capacity (see e.g. [Ran]). Put $\widetilde{E}=\bigcap_{j} U_{j}$. Then $c(\widetilde{E})=0$ (so $\widetilde{E}$ is polar), $\widetilde{E}$ is a $G_{\delta}$ set, $\widetilde{E} \supset E$, and $z_{0} \notin \widetilde{E}$. Hence, $\widetilde{E}$ is complete polar and $z_{0} \notin E_{\mathbb{C}}^{*}$.

Recall the following result (see [Anc]). 
THEOREM 8 (Ancona's theorem). Let $K$ be a compact non-polar set in $\mathbb{C}$. Then for any $\varepsilon>0$ there exists a compact set $K^{\prime} \subset K$ such that $c\left(K \backslash K^{\prime}\right)<\varepsilon$ and $K^{\prime}$ is regular at any point of itself.

As a corollary we get the following useful result.

Corollary 9. Let $E$ be a Borel set in $\mathbb{C}$. Assume that $E$ is non-polar. Then there exists a sequence of compact sets $K_{1} \subset K_{2} \subset \cdots \subset E$, regular at any point of each of them, and a polar Borel set $P$ such that $E=P \cup \bigcup_{j} K_{j}$.

Proof. First note that there exists an $F_{\sigma}$ set $E_{1}$ and a polar set $P_{1}$ so that $E=E_{1} \cup P_{1}$. We have $E_{1}=\bigcup_{j} \widetilde{K}_{j}$, where $\widetilde{K}_{j}$ is an increasing sequence of compact sets. Now, it suffices to use Theorem 8.

3. Proof of the main result. Recall the following localization principle [EdWi3].

THEOREM 10. Let $\Omega \subset \mathbb{C}^{n}$ be an open set and let $E$ be an $F_{\sigma}$ pluripolar subset of $\Omega$. Then for any open set $\Omega^{\prime} \Subset \Omega$ and any open set $U$ such that $\partial U \cap E_{\Omega}^{*}=\emptyset$ we have

$$
\omega\left(z, E \cap U \cap \Omega^{\prime}, \Omega^{\prime}\right)=\omega\left(z, E \cap U \cap \Omega^{\prime}, U \cap \Omega^{\prime}\right), \quad z \in U \cap \Omega^{\prime} .
$$

Proof of Theorem 3. Let $p \in f\left(E_{\Omega}^{*}\right) \backslash f(E)$ and let $z_{0} \in f^{-1}(p) \cap E_{\Omega}^{*}$. Put $F=\mathbb{C} \backslash f\left(E_{\Omega}^{*}\right)$. Then $F$ is Borel $\left(G_{\delta}\right)$. Assume that $F$ is not thin at $p$. Hence, there exists a sequence of compact sets $K_{1} \subset K_{2} \subset \cdots \subset F$, regular at any point of each of them, and a polar Borel set $P$ such that $F \backslash\{p\}=P \cup \bigcup_{j} K_{j}$.

Put $U_{j}=f^{-1}\left(\mathbb{C} \backslash K_{j}\right) \cap U$. Since $E$ is an $F_{\sigma}$ set, there exists a sequence of compact sets $E_{1} \subset E_{2} \subset \cdots \subset E$ such that $E=\bigcup_{j} E_{j}$. Then $E_{\Omega}^{*}=$ $\bigcup_{j}\left(E_{j}\right)_{\Omega}^{*}$. Hence, $p \in \bigcup_{j} f\left(\left(E_{j}\right)_{\Omega}^{*}\right)$. Put $L_{j}=f\left(E_{j}\right)$.

First, assume that $f(E)$ is non-polar. Then without loss of generality, we may assume that $L_{1}$ is non-polar.

Fix a hyperconvex domain $\Omega^{\prime} \Subset \Omega$. We want to estimate $\omega\left(z_{0}, E_{j} \cap\right.$ $\left.\Omega^{\prime}, \Omega^{\prime}\right)$. By the localization principle we have

$$
\omega\left(z_{0}, E_{j} \cap \Omega^{\prime}, \Omega^{\prime}\right)=\omega\left(z_{0}, E_{j} \cap \Omega^{\prime}, \Omega^{\prime} \cap U_{k}\right) \leq \omega\left(p, L_{j}, \widehat{\mathbb{C}} \backslash K_{k}\right) .
$$

We claim that

$$
\lim _{k \rightarrow \infty} \omega\left(p, L_{j}, \widehat{\mathbb{C}} \backslash K_{k}\right)=0 .
$$

Fix $j \geq 1$. For each natural number $k$ we let $D_{k}$ be the connected component of $\widehat{\mathbb{C}} \backslash K_{k}$ which contains $p$. We have

$$
\omega\left(p, L_{j}, \widehat{\mathbb{C}} \backslash K_{k}\right)=\omega\left(p, L_{j} \cap D_{k}, D_{k}\right) .
$$

Note that $D_{k}$ is a regular domain (see [Ran, Theorem 4.2.4]). Put $h_{k}(z)=$ $\omega\left(z, L_{j} \cap D_{k}, D_{k}\right)$. Then $h_{k}$ is a harmonic function on $D_{k}$. Moreover, it extends subharmonically to $\widehat{\mathbb{C}} \backslash L_{j}$ (we put $h_{k}=0$ on $\widehat{\mathbb{C}} \backslash D_{k}$ ). Hence 
$h(z)=\lim _{k \rightarrow \infty} h_{k}(z)$ is non-negative and subharmonic on $\widehat{\mathbb{C}} \backslash L_{j}$ (being the decreasing limit of a sequence of subharmonic functions). Moreover, $h=0$ on $\bigcup_{k} K_{k}$. Since $\bigcup_{k} K_{k}$ is non-thin at $p, p$ is an accumulation point of $\bigcup_{k} K_{j}$ and $h(p)=0$. Hence, we have proved (3.2).

So, we have $\omega\left(z_{0}, E_{j} \cap \Omega^{\prime}, \Omega^{\prime}\right)=0$. Hence, $z_{0} \notin\left(E_{j} \cap \Omega^{\prime}\right)_{\Omega^{\prime}}^{-}$. Since $\Omega^{\prime} \Subset \Omega$ is an arbitrary hyperconvex domain, we get $z_{0} \notin\left(E_{j}\right)_{\Omega}^{*}$ and $z_{0} \notin \bigcup_{j}\left(E_{j}\right)_{\Omega}^{*}$. But we know that $z_{0} \in f^{-1}(p) \cap E_{\Omega}^{*}$. A contradiction.

Now, assume that $f(E)$ is polar. Note that $f\left(E_{\Omega}^{*}\right) \subset f(E)_{\mathbb{C}}^{*}=f(E)$.

Assume that $f(E)$ is open in the fine topology. Take $p \in f\left(E_{\Omega}^{*}\right)$. Note that there are two cases: $p \in f\left(E_{\Omega}^{*}\right) \backslash f(E)$ and $p \in f(E)$. In both cases we see that $\mathbb{C} \backslash f\left(E_{\Omega}^{*}\right)$ is thin at $p$.

Proof of Corollary 3. Note that $\pi_{1}\left(\Gamma_{f}(D)\right)=D$ is open and, therefore, open in the fine topology. If $f$ is non-constant then $\pi_{2}\left(\Gamma_{f}(D)\right)=f(D)$ is also open.

4. Example. Note that in Corollary 3 we cannot state, in general, that $\pi_{1}\left(\left(\Gamma_{f}(D)\right)_{\mathbb{C}^{2}}^{*}\right)$ is open. Indeed, take $a_{n}=1 / n$ and $c_{n}=e^{-n^{2}} / n^{2}, n \in \mathbb{N}$. Put

$$
f(z)=\sum_{n=1}^{\infty} \frac{c_{n}}{z-a_{n}} .
$$

Note that $f$ is a holomorphic function on the domain $D=\mathbb{C} \backslash\left\{a_{n}: n \in \mathbb{C}\right\}$ $\cup\{0\}$. By [EdWi1], $\left(\Gamma_{f}(D)\right)_{\mathbb{C}^{2}}^{*}=\Gamma_{f}(D) \cup\{(0, f(0))\}$. So, $\pi_{1}\left(\left(\Gamma_{f}(D)\right)_{\mathbb{C}^{2}}^{*}\right)=$ $D \cup\{0\}$.

\section{References}

[Anc] A. Ancona, Démonstration d'une conjecture sur la capacité et l'effilement, C. R. Acad. Sci. Paris 297 (1983), 393-395.

[Bre] M. Brelot, Éléments de la théorie classique du potentiel, Les cours de Sorbonne, Paris, 1958.

[Edi] A. Edigarian, Pluripolar hulls and holomorphic coverings, Israel J. Math. 130 (2002), 77-92,

[EdWi1] A. Edigarian and J. Wiegerinck, The pluripolar hull of the graph of a holomorphic function with polar singularities, Indiana Univ. Math. J. 52 (2003) 1663-1680.

[EdWi2] - - - Graphs that are not complete pluripolar, Proc. Amer. Math. Soc. 131 (2003), 2459-2465.

[EdWi3] - - - Determination of the pluripolar hull of graphs of certain holomorphic functions, Ann. Inst. Fourier (Grenoble) 54 (2004), 2085-2104.

[Edl] T. Edlund, Complete pluripolar curves and graphs, preprint, Dept. Math., Uppsala Univ., 2004.

[E-J1] T. Edlund and B. Jöricke, The pluripolar hull of a graph and fine analytic continuation, arXiv:math.CV/0405025 (2004). 
[E-J2] T. Edlund and B. Jöricke, The pluripolar hull of a graph and fine analytic continuation, Ark. Mat., to appear.

[Jos] B. Josefson, On the equivalence between locally polar and globally polar sets for plurisubharmonic functions on $\mathbb{C}^{n}$, ibid. 16 (1978), 109-115.

[LePo] N. Levenberg and E. A. Poletsky, Pluripolar hulls, Michigan Math. J. 46 (1999), 151-162.

[LeMaPo] N. Levenberg, G. Martin and E. A. Poletsky, Analytic disks and pluripolar sets, Indiana Univ. Math. J. 41 (1992), 515-532.

[Ran] Th. Ransford, Potential Theory in the Complex Plane, Cambridge Univ. Press, 1994.

[Sad] A. Sadullaev, Plurisubharmonic measures and capacities on complex manifolds, Uspekhi Mat. Nauk 36 (1981), no. 4, 53-105 (in Russian).

[Sic] J. Siciak, Pluripolar sets and pseudocontinuation, in: Complex Analysis and Dynamical Systems II (Nahariya, 2003), Amer. Math. Soc., Contemp. Math., to appear.

[Wie1] J. Wiegerinck, The pluripolar hull of $\left\{w=e^{-1 / z}\right\}$, Ark. Mat. 38 (2000), 201-208.

[Wie2] - Graphs of holomorphic functions with isolated singularities are complete pluripolar, Michigan Math. J. 47 (2000), 191-197.

[Zer] A. Zeriahi, Ensembles pluripolaires exceptionnels pour la croissance partielle des fonctions holomorphes, Ann. Polon. Math. 50 (1989), 81-91.

[Zwo] W. Zwonek, A note on pluripolar hulls of graphs of Blaschke products, Potential Anal. 22 (2005), 195-206.

Armen Edigarian

Institute of Mathematics

Jagiellonian University

Reymonta 4

30-059 Kraków, Poland

E-mail: Armen.Edigarian@im.uj.edu.pl 